\title{
Common Fixed Points for Faintly Compatible Mappings
}

\author{
N. Chandra*, Mahesh C. Joshi and Narendra K. Singh
}

ABSTRACT. In this paper, we obtain a generalized common fixed point theorem for four mappings using the conditions of non-compatibility and faint compatibility.

\section{Introduction AND PRELIMINARIES}

Generalizing Banach contraction principle, Jungck [9] initiated the study of common fixed points for a pair of commuting mappings satisfying contractive type conditions. In 1982, Sessa [14] introduced the weaker notion of commutativity which is generally known as Weak Commutativity and established some interesting results on the existence of common fixed points for the pair of mappings. Further, Jungck [10] generalized the concept of weak commutativity by introducing the notion of compatible mappings. Throughout this section $(f, g)$ denotes a pair of mapping on a metric space $X$.

Definition 1.1 ([10]). The pair of mappings $(f, g)$ is said to be compatible iff $\lim _{n \rightarrow \infty} d\left(f g x_{n}, g f x_{n}\right)=0$, whenever $\left\{x_{n}\right\}$ is a sequence in $X$ such that $\lim _{n \rightarrow \infty} f x_{n}=\lim _{n \rightarrow \infty} g x_{n}=t$ for some $t \in X$.

Definition $1.2([10])$. The pair $(f, g)$ is said to be non-compatible if there exists a sequence $\left\{x_{n}\right\}$ in $X$ such that $\lim _{n \rightarrow \infty} f x_{n}=\lim _{n \rightarrow \infty} g x_{n}=t$ for some $t \in X$ but $\lim _{n \rightarrow \infty} d\left(f g x_{n}, g f x_{n}\right)$ is either non-zero or non-existent.

Again in 1996, Jungck [8] generalized the the concept of compatibility by introducing weakly compatible mappings.

Definition $1.3([8])$. The pair $(f, g)$ is said to be weakly compatible if the pair commutes on the set of coincidence points, i.e., $f g x=g f x$ whenever $f x=g x$ for some $x \in X$.

Al-Thagafi and Shahzad [2] introduced the concept of occasionally weakly compatible mappings by weakened the notion of weakly compatible mappings.

2010 Mathematics Subject Classification. 47H10, 54H25.

Key words and phrases. Common fixed points, compatibility, faint compatibility.

*Author thanks UGC, New Delhi for support under RGNF. 
Definition $1.4([2])$. The pair $(f, g)$ is said to be occasionally weakly compatible if there exists a coincidence point $x \in X$ such that $f x=g x$ implies $f g x=g f x$.

In 2010, Pant et al. [12] redefined the concept of occasionally weakly compatible mappings by introducing conditional commutativity.

Definition 1.5 ([12]). The pair $(f, g)$ is said to be conditionally commuting if the pair commutes on a nonempty subset of the set of coincidence points whenever the set of coincidences is nonempty.

Again, Pant et al. [13] gave the concept of conditional compatibility which is indepedent of compatibility condition and proved that in case of existence of unique common fixed/coincidenence point, conditional compatibility can not be reduced to the compatibility condition. Further, they also proved that conditional compatibility need not imply commutativity at the coincidence points.

Definition $1.6([13])$. The pair $(f, g)$ is said to be conditionally compatible iff whenever the set of sequences $\left\{x_{n}\right\}$ satisfying $\lim _{n \rightarrow \infty} f x_{n}=\lim _{n \rightarrow \infty} g x_{n}$ is nonempty, there exists a sequence $\left\{y_{n}\right\}$ such that

$$
\lim _{n \rightarrow \infty} f y_{n}=\lim _{n \rightarrow \infty} g y_{n}=t \text { and } \lim _{n \rightarrow \infty} d\left(f g y_{n}, g f y_{n}\right)=0 .
$$

Over the last two decades, there are a number of common fixed/coincidence point theorems for the pair of mappings under different contractive conditions with compatibility and its weaker versions imposed on the mappings (for more details, see $[4,5,6,7,1,3]$ and refenences therein).

In a recent work, Bisht and Shahzad [3] gave a new notion of conditionally compatible mappings in a slighty different settings and named it as faintly compatible mappings.

Definition $1.7([3])$. The pair $(f, g)$ is said to be faintly compatible iff $(f, g)$ is conditionally compatible and $(f, g)$ commutes on a nonempty subset of coincidence points whenever the set of coincidences is nonempty.

Bisht et al. [3] proved some interesting common fixed point theorems using the concept of faintly compatible mappings on non-complete metric spaces under defferent contractive conditions. Complementing the work of Bisht et al. [3], we give following examples for the comparative discussions on the above concepts.

(i) Compatibilty implies faint compatibility but converse may not be true.

Example 1.1. Let $X=[2,4]$ and $d$ be the usual metric on $X$. Define self mappings $f$ and $g$ on $X$ as follows:

$$
f(x)=\left\{\begin{array}{ll}
2 & \text { if } x=2 \text { or } x>3 \\
x+1 & \text { if } 2<x \leq 3
\end{array} \text { and } g(x)= \begin{cases}2 & \text { if } x=2 \\
\frac{x+4}{2} & \text { if } 2<x \leq 3 \\
\frac{x+1}{2} & \text { if } x>3\end{cases}\right.
$$


In this example, $f$ and $g$ are faintly compatible but not compatible. For if, we consider the constant sequence $\left\{x_{n}=2\right\}$, then $f$ and $g$ are faintly compatible. On the other hand, if we choose a sequence $\left\{y_{n}=3+\frac{1}{n}\right\}$, then $\lim _{n \rightarrow \infty} f y_{n}=\lim _{n \rightarrow \infty} g y_{n}=2$ and $\lim _{n \rightarrow \infty} d\left(f g y_{n}, g f y_{n}\right)=1(\neq 0)$. Hence $f$ and $g$ are not compatible.

(ii) Faint compatibility and non-compatibility are independent concepts.

Example 1.2. Let $X=[2,8]$ and $d$ be the usual metric on $X$. Define self mappings $f$ and $g$ on $X$ as follows:

$$
f(x)=\left\{\begin{array}{ll}
6 & \text { if } 2 \leq x \leq 4 \\
2 & \text { if } x>4
\end{array} \text { and } g(x)= \begin{cases}2 & \text { if } 2 \leq x<4 \\
x-2 & \text { if } x \geq 4\end{cases}\right.
$$

In this example, $f$ and $g$ are non-compatible but not faintly compatible. To see this, we consider a sequence $\left\{x_{n}=4+\frac{1}{n}\right\}$, then $\lim _{n \rightarrow \infty} f x_{n}=2=\lim _{n \rightarrow \infty} g x_{n}$ but $\lim _{n \rightarrow \infty} d\left(f g x_{n}, g f x_{n}\right)=4$. So, $f$ and $g$ are non-compatible.

Example 1.3. Let $X=[1, \infty)$ and let $d$ be the usual metric on $X$. Define self mappings $f$ and $g$ on $X$ as follows:

$$
f(x)=x \quad \forall x \in X \quad \text { and } \quad g(x)=3 x-2 \quad \forall x \in X .
$$

In this one, $f$ and $g$ are faintly compatible but not non-compatible.

(iii) Weakly compatible implies faint compatibility, but converse is not true in general.

Example 1.4. Let $X=\left[0, \frac{2}{3}\right]$ with the usual metric $d$. Define self mappings $f$ and $g$ on $X$ as follows:

$$
f(x)=\frac{1}{3}-\left|\frac{1}{3}-x\right| \quad \text { and } \quad g(x)=\frac{2-3 x}{9} .
$$

In this example the mappings $f$ and $g$ are faintly compatible but not weakly compatible. To see this, we take a constant sequence $\left\{x_{n}=\frac{1}{12}\right\}$ and they are commuting at the coincidence point $x=\frac{1}{12}$. On the other hand, $f$ and $g$ do not commute at the coincidence point $x=\frac{2}{3}$, hence they are not weakly compatible.

(iv) Occasionally weakly compatible implies faintly compatible but the converse may not be true.

Example 1.5. Let $X=[0, \infty)$ with usual metric $d$ on $X$. Define self mappings $f$ and $g$ on $X$ as follows:

$$
f(x)=\frac{x}{2} \quad \forall x \in X \quad \text { and } \quad g(x)=\frac{x+3}{2} \quad \forall x \in X .
$$

In this example, mappings $f$ and $g$ are trivially faintly compatible but not occasionally compatible. 
In one of the interesting paper, Jungck [11] established a common fixed point theorem for four mappings in a complete metric space. Now, we prove our main result for the existence of common fixed point for four mappings in a non-complete metric space using the concept of faintly compatible mappings which is analogous to the result of Jungck [11].

\section{Main Results}

Theorem 2.1. Let $A, B, S$ and $T$ be continuous self mappings of a metric space $(X, d)$. Suppose

(i) pairs $(A, S)$ and $(B, T)$ are non-compatible and faintly compatible,

(ii) $A X \subset T X$ and $B X \subset S X$.

If there exists $k \in(0,1)$ such that

$$
\begin{array}{r}
d(A x, B y) \leq k \max \{d(A x, S x), d(B y, T y), d(S x, T y), \\
\left.\frac{1}{2}[d(A x, T y)+d(B y, S x)]\right\}
\end{array}
$$

for $x, y \in X$. Then there is a unique point $z \in X$ such that $A z=B z=$ $S z=T z=z$.

Proof. As the pair $(A, S)$ is non-compatible, then there exists a sequence $\left\{x_{n}\right\}$ in $X$ such that $\lim _{n \rightarrow \infty} A x_{n}=\lim _{n \rightarrow \infty} S x_{n}=t$ for some $t \in X$ but $\lim _{n \rightarrow \infty} d\left(A S x_{n}, S A x_{n}\right)$ is either non-zero or non-existent. Since $A$ and $S$ are faintly compatible and $\lim _{n \rightarrow \infty} A x_{n}=\lim _{n \rightarrow \infty} S x_{n}=t$, there exists a sequence $\left\{z_{n}\right\}$ in $X$ satisfying $\lim _{n \rightarrow \infty} A z_{n}=\lim _{n \rightarrow \infty} S z_{n}=u($ say $)$ such that

$$
\lim _{n \rightarrow \infty} d\left(A S z_{n}, S A z_{n}\right)=0 .
$$

Further, since $A$ is continuous, $\lim _{n \rightarrow \infty} A A z_{n}=A u$ and $\lim _{n \rightarrow \infty} A S z_{n}=$ $A u$. These last three limits together imply $\lim _{n \rightarrow \infty} S A z_{n}=A u$. The inclusion $A X \subset T X$ implies that $A u=T v$ for some $v \in X$ and $\lim _{n \rightarrow \infty} A A z_{n}=$ $T v, \lim _{n \rightarrow \infty} S A z_{n}=T v$.

Similarly, non-compatibility of the pair $B, T$ implies that there exists a sequence $\left\{y_{n}\right\}$ in $X$ such that $\lim _{n \rightarrow \infty} B y_{n}=\lim _{n \rightarrow \infty} T y_{n}=t^{\prime}$ for some $t^{\prime} \in X$ but $\lim _{n \rightarrow \infty} d\left(B T y_{n}, T B y_{n}\right)$ is either non-zero or non-existent. Now faint compatibility of $B$ and $T$ will imply that there exists a sequence $\left\{w_{n}\right\}$ in $X$ satisfying $\lim _{n \rightarrow \infty} B w_{n}=\lim _{n \rightarrow \infty} T w_{n}=u^{\prime}$ (say) such that

$$
\lim _{n \rightarrow \infty} d\left(B T w_{n}, T B w_{n}\right)=0 .
$$

Again, $B$ is continuous so $\lim _{n \rightarrow \infty} B B w_{n}=B u^{\prime}$ and $\lim _{n \rightarrow \infty} B T w_{n}=B u^{\prime}$. These last three limits together imply $\lim _{n \rightarrow \infty} T B w_{n}=B u^{\prime}$. The inclusion $B X \subset S X$ implies that $B u^{\prime}=S v^{\prime}$ for some $v^{\prime} \in X$ and $\lim _{n \rightarrow \infty} B B w_{n}=$ $S v^{\prime}, \lim _{n \rightarrow \infty} T B w_{n}=S v^{\prime}$. 
Using the condition (1), we get

$$
\begin{aligned}
d\left(u, u^{\prime}\right)= & \lim _{n \rightarrow \infty} d\left(A z_{n}, B w_{n}\right) \\
& \leq k \lim _{n \rightarrow \infty} \max \left\{d\left(A z_{n}, S z_{n}\right), d\left(B w_{n}, T w_{n}\right), d\left(S z_{n}, T w_{n}\right),\right. \\
& \frac{1}{2}\left[d\left(A z_{n}, T w_{n}\right)+d\left(B w_{n}, S z_{n}\right)\right\} \\
& =k \max \left\{d\left(u, u^{\prime}\right), d\left(u^{\prime}, u^{\prime}\right), d\left(u, u^{\prime}\right), \frac{1}{2}\left[d\left(u, u^{\prime}\right)+d\left(u^{\prime}, u\right)\right]\right\} .
\end{aligned}
$$

Thus

$$
d\left(u, u^{\prime}\right) \leq k d\left(u, u^{\prime}\right) \quad \Rightarrow \quad d\left(u, u^{\prime}\right)=0 \quad \Rightarrow \quad u=u^{\prime}
$$

So, $A u=T v$ and $B u=S v^{\prime}$.

Now, $\lim _{n \rightarrow \infty} A z_{n}=\lim _{n \rightarrow \infty} S z_{n}=\lim _{n \rightarrow \infty} B w_{n}=\lim _{n \rightarrow \infty} T w_{n}=u$.

Continuity of $S$ and $T$ together with conditions (2) and (3) imply

$$
\lim _{n \rightarrow \infty} S S z_{n}=\lim _{n \rightarrow \infty} S A z_{n}=S u \Rightarrow \lim _{n \rightarrow \infty} S S z_{n}=\lim _{n \rightarrow \infty} A S z_{n}=S u,
$$

and $\lim _{n \rightarrow \infty} T B w_{n}=\lim _{n \rightarrow \infty} T T w_{n}=T u \Rightarrow \lim _{n \rightarrow \infty} T T w_{n}=\lim _{n \rightarrow \infty} B T w_{n}=T u$.

Now,

$d\left(A S z_{n}, B T w_{n}\right) \leq k \max \left\{d\left(A S z_{n}, S S z_{n}\right), d\left(B T w_{n}, T T w_{n}\right), d\left(S S z_{n}, T T w_{n}\right)\right.$,

$$
\frac{1}{2}\left[d\left(A S z_{n}, T T w_{n}\right)+d\left(B T w_{n}, S S z_{n}\right)\right\} .
$$

Taking $n \rightarrow \infty$, we get

$d(S u, T u) \leq k \max \left\{d(S u, S u), d(T u, T u), d(S u, T u), \frac{1}{2}[d(S u, T u)+d(T u, S u)]\right\}$

$$
\begin{aligned}
& \Rightarrow \quad d(S u, T u) \leq k d(S u, T u) \Rightarrow d(S u, T u)=0 \\
& \Rightarrow S u=T u .
\end{aligned}
$$

Also,

$$
\begin{aligned}
d(A u, T u)= & \lim _{n \rightarrow \infty} d\left(A u, B T w_{n}\right) \\
& \leq \max \lim _{n \rightarrow \infty}\left\{d(A u, S u), d\left(B T w_{n}, T T w_{n}\right), d\left(S u, T T w_{n}\right),\right. \\
& \frac{1}{2}\left[d\left(A u, T T w_{n}\right)+d\left(B T w_{n}, S u\right)\right\} \\
\Rightarrow d(A u, T u) \leq & k \max \{d(A u, S u), d(T u, T u), d(S u, T u), \\
& \left.\frac{1}{2}[d(A u, T u)+d(T u, S u)]\right\} \\
\Rightarrow d(A u, T u) \leq & k \max \left\{d(A u, T u), \frac{1}{2} d(A u, T u)\right\} \\
\Rightarrow d(A u, T u)= & 0
\end{aligned}
$$




$$
\Rightarrow \quad A u=T u .
$$

Using (2.1) with $x=y=u$, we get

$$
\begin{aligned}
& d(A u, B u) \leq k \max \{d(A u, S u), d(B u, T u), d(S u, T u), \\
&\left.\frac{1}{2}[d(A u, T u)+d(B u, S u)]\right\} \\
& \Rightarrow d(A u, B u) \leq k \max \{d(B u, A u), \\
&\left.\frac{1}{2} d(B u, A u)\right\} \\
& \Rightarrow d(A u, B u)= 0 \\
& \Rightarrow A u=B u .
\end{aligned}
$$

From (4), (5) and (6), we have $A u=B u=S u=T u$. In fact, $u$ is a common fixed point $A, B, S$ and $T$. To see this,

$$
\begin{aligned}
d(u, B u)= & \lim _{n \rightarrow \infty} d\left(A z_{n}, B u\right) \\
& \leq k \max \lim _{n \rightarrow \infty}\left\{d\left(A z_{n}, S z_{n}\right), d(B u, T u), d\left(S z_{n}, T u\right),\right. \\
& \left.\frac{1}{2}\left[d\left(A z_{n}, T u\right)+d\left(B u, S z_{n}\right)\right]\right\} \\
\Rightarrow d(u, B u) \leq & k \max \left\{d(u, T u), \frac{1}{2}[d(u, T u)+d(B u, u)]\right\} \\
\Rightarrow d(u, B u) \leq & k \max \left\{d(u, B u), \frac{1}{2} d(u, B u)\right\} \\
\Rightarrow d(u, B u)= & 0 \Rightarrow B u=u .
\end{aligned}
$$

Hence, $A u=B u=S u=T u=u$.

For the uniqueness of the common fixed point, let $w$ be another common fixed point of $A, B, S$ and $T$, i.e., $A w=B w=S w=T w=w$. We have

$$
\begin{gathered}
d(u, w)=A(A u, B w) \leq k \max \{d(A u, B u), d(B w, T w), d(S u, T w), \\
\left.\frac{1}{2}[d(A u, T w)+d(B w, S u)]\right\} \\
\Rightarrow d(u, w) \leq k d(u, w) \Rightarrow u=w .
\end{gathered}
$$

Remark 2.1. In Theorem 2.1, taking $(A, S)$ and $(B, T)$ as compatible pairs of mappings and metric space as complete, we get the result of Jungck [11].

Now, we give an example in the support of our main result.

Example 2.1. Let $X=[0,10]$ with usual metric $d$ on $X$. Define self mappings $A, B, S$ and $T$ on $X$ as follows:

$$
A x=\left\{\begin{array}{ll}
5 & \text { if } x \leq 5 \\
10-x & \text { if } x>5
\end{array} \quad B x= \begin{cases}\frac{3 x+10}{5} & \text { if } x \leq 5 \\
10-x & \text { if } x>5\end{cases}\right.
$$




$$
S x=\left\{\begin{array}{ll}
\frac{-3 x+40}{5} & \text { if } x \leq 5 \\
10-x & \text { if } x>5
\end{array} \quad \text { Tx=10-x } \quad \forall x \in X .\right.
$$

In this example, pairs $(A, S)$ and $(B, T)$ on $X$ are faintly compatible mappings. For this, we consider the constant sequence $\left\{x_{n}=5\right\}$ and $A S(5)=S A(5)$. Also, pairs $(A, S)$ and $(B, T)$ are non-compatible mappings. To see this, consider the sequence $\left\{x_{n}\right\}$ in $X$ such that $\lim _{n \rightarrow \infty} x_{n}=10$, then

$$
\lim _{n \rightarrow \infty} A x_{n}=\lim _{n \rightarrow \infty} S x_{n}=\lim _{n \rightarrow \infty} B x_{n}=\lim _{n \rightarrow \infty} T x_{n}=0
$$

and

$$
\lim _{n \rightarrow \infty} d\left(A S x_{n}, S A x_{n}\right) \neq 0, \quad \lim _{n \rightarrow \infty} d\left(B T x_{n}, T B x_{n}\right) \neq 0 .
$$

It can be verified that the mappings $A, B, S$ and $T$ on $X$ are satisfying the condition (1) with $k=\frac{3}{8}$ and 5 is the only common fixed point of $A, B, S$ and $T$.

Taking $A=B$ and $S=T$ in Theorem 2.1, we obtain following corollary.

Corollary 2.1. Let $A, S$ be self mappings of a metric space $(X, d)$. Suppose

(i) $A$ and $S$ are continuous,

(ii) pairs $(A, S)$ is non-compatible faintly compatible,

(iii) $A X \subset S X$.

If there exists $k \in(0,1)$ such that

$$
\begin{array}{r}
d(A x, A y) \leq k \max \{d(A x, S x), d(A y, S y), d(S x, S y), \\
\left.\frac{1}{2}[d(A x, S y)+d(A y, S x)]\right\}
\end{array}
$$

for $x, y \in X$. Then $A$ and $S$ have unique common fixed point in $X$.

Remark 2.2. Observe that Corollary 2.1 is a generalization of the result due to Bisht et al. ([3], Theorem 2.1) .

Corollary 2.2. Let $A, B, S$ and $T$ be continuous self mappings of a metric space $(X, d)$. Suppose the pairs $(A, S)$ and $(B, T)$ are non-compatible faintly compatible and $A X \subset T X$ and $B X \subset S X$. If there exists $k \in(0,1)$ such that any of the following inequalities holds

(i) $d(A x, B y) \leq k \max \{d(A x, S x), d(B y, T y)\}$

(ii) $d(A x, B y) \leq k \max \{d(A x, S x), d(B y, T y), d(S x, T y)\}$

(iii) $d(A x, B y) \leq k \max \left\{d(A x, S x), d(B y, T y), \frac{1}{2}[d(A x, T y)+d(B y, S x)]\right\}$ for all $x, y \in X$. Then there is a unique point $z \in X$ such that $A z=B z=$ $S z=T z=z$.

If we consider $A=T$ and $B=S$ in Theorem 2.1, we have following corollary. 
Corollary 2.3. Let $A$ and $B$ be continuous self mappings of a metric space $(X, d)$, the pair $(A, B)$ be non-compatible faintly compatible and $A X \subset B X$. If there exists $k \in(0,1)$ such that

$$
\begin{array}{r}
d(A x, B y) \leq k \max \{d(A x, B x), d(A y, B y), d(B x, A y), \\
\left.\frac{1}{2}[d(A x, A y)+d(B x, B y)]\right\}
\end{array}
$$

for all $x, y \in X$. Then $A$ and $B$ have a unique common fixed point in $X$.

Taking $T=S=I$ (identity map) in Theorem 2.1, we obtain following result as corollary.

Corollary 2.4. Let $A$ and $B$ be continuous self mappings of a metric space $(X, d)$, the pair $(A, B)$ be non-compatible faintly compatible and $A X \subset B X$. If there exists $k \in(0,1)$ such that

$d(A x, B y) \leq k \max \left\{d(x, y), d(x, A x), d(y, B y), \frac{1}{2}[d(y, A x)+d(x, B y)]\right\}$

for all $x, y \in X$. Then $A$ and $B$ have a unique common fixed point in $X$.

\section{REFERENCES}

[1] M. Abbas, Lj. Ćirić, B. Damjanović, M. A. Khan, Coupled coincidence and common fixed point theorems for hybrid pair of mappings, Fixed Point Theory Appl., (2012). doi: 10.1186/1687-1812-2012-4.

[2] M. A. Al-Thagafi, N. shahzad, Generalized l-nonexpansive self maps and invariant approximations, Act. Math. Sin., 24(2008), 867-876.

[3] R. K. Bisht, N. Shahzad, Faintly compatible mappings and common fixed points, Fixed Point Theory Appl., (2013). doi: 10.1186/1687-1812-2013-156.

[4] Lj. Ćirić, B. Samet, C. Vetro, Common fixed point theorems for families of occasionally weakly compatible mappings, Math. Comput. Model., 53(5-6)(2011), 631-636.

[5] Lj. Ćirić, B. Samet, N. Cakić, B. Damjanović, Generalized $(\psi, \omega)$-weak nonlinear contractions in ordered K-metric spaces, Comput. Math. Appl., 62(2011), 3305-3316.

[6] Lj. Ćirić, A. Razani, S. Radenović, J. S. Ume, Common fixed point theorems for families of weakly compatible maps, Comput. Math. Appl., 55(11)(2008), 2533-2543.

[7] Lj. Ćirić, N. T. Nikolić, J. S. Ume, Common fixed point theorems for weakly compatible quasi contraction mappings, Acta Math. Hung., 113(4)(2006), 257-267.

[8] G. Jungck, Common fixed points for non-continuous non-self maps on non-metric spaces, Far East J. Math. Sci., 4(1996), 199-215.

[9] G. Jungck, Commuting mappings and fixed points, Amer. Math. Monthly, 83(1976), 261-263.

[10] G. Jungck, Compatible mappings and common fixed points, Int. J. Math. Math. Sci., 9(4)(1986), 771-779.

[11] G. Jungck, Compatible mappings and common fixed points (2), Int. J. Math. Math. Sci., 11(2)(1988), 285-288. 
[12] V. Pant, R. P. Pant, Common fixed points of conditionally commuting maps, Fixed Point Theory, 1(2010), 113-118.

[13] R. P. Pant, R. K. Bisht, Occasionally weakly compatible mappings and fixed points, Bull. Belg. Math. Soc. Simon Stevin, 19(2012), 655-661.

[14] S. Sessa, On a weak commutativity condition of mappings in fixed point consideration, Publ. Inst. Math., 32(1982), 149-153.

N. Chandra

Department of Mathematics

D.S.B. Campus, Kumaun University

263002 Nainital

INDIA

E-mail address: cnaveen329@gmail.com

Mahesh C. Joshi

Department of Mathematics

D. S. B. Campus, Kumaun University

263002 NAINITAL

INDIA

E-mail address: mcjoshi69@gmail.com

NARENDRA K. Singh

Department of Mathematics

D. S. B. Campus, Kumaun University

263002 NAINITAL

INDIA

E-mail address: nsijwali@gmail.com 